RUHUNA JOURNAL OF SCIENCE

Vol 12 (1): 26-39, June 2021

eISSN: $2536-8400$

(c) Faculty of Science

http://doi.org/10.4038/rjs.v12i1.98

\title{
Forest resources as ecotourism attraction: Cross River National Park, Nigeria
}

\author{
Bukola O. Adetola ${ }^{* 1}$, Abideen A. Alarape ${ }^{2}$ and Ibukun A. Ayodele ${ }^{2}$ \\ ${ }^{1}$ Department of Ecotourism and Wildlife Management, Federal University of Technology, Akure, Ondo \\ State, Nigeria \\ ${ }^{2}$ Department of Wildlife and Ecotourism Management, University of Ibadan, Oyo State, Nigeria \\ *Correspondence: boadetola@futa.edu.ng; (iD ORCID: https://orcid.org/0000-0002-1675-3344
}

Received: $5^{\text {th }}$ June 2020, Revised: $2^{\text {th }}$ April 2021, Accepted: $10^{\text {th }}$ June 2021

\begin{abstract}
Cross River National Park (CRNP) in Nigeria with its natural resources of tourist significance can help to foster a culture of conservation and recreation among the public. This study identified ecotourism attractions in CRNP aiming to promote its sustainable use and to enhance its planning as a haven for ecotourism in Nigeria. A field survey of ecotourism resources was conducted in the park during 2010-2014. Fauna resources were identified along the $5 \mathrm{~km}$ transects that covered existing trails and jeep tracks in both dry and wet seasons. Quadrat method (15 sample plots of 50 $\mathrm{m} \times 20 \mathrm{~m}$ ) was used for the vegetation assessment and Shannon-Weiner Diversity Index $(H)$ and descriptive statistics were calculated. Findings revealed that the rainforest, game viewing, birdwatching, mountain climbing, camping, rock formation, village tour, botanical garden tour and water recreation as core ecotourism attractions in CRNP. Fifteen wild animal species belonging to nine families were observed (endangered 01, least concerned 12, near threatened 01, vulnerable 01). Wild animals included drill monkey Mandrillus leucophaecus (endangered), elephant Loxodonta africana cyclotis (vulnerable), bat Eidolon helvum (near threatened), and bare-headed rock fowl Picarthates oreas (endemic) which are "ecostars" and "a must see" that warrant a visit to the park. Other animals were least concerned. A total of 81 tree species belonging to 26 families were identified. Shannon-Wiener Diversity index (H') of 3.88 and 3.84 were recorded for Oban and Okwango divisions respectively. The most dominant family in Oban was Leguminosae (12 species) and in Okwango, it was Apocynaceae (10 species). Managing and promoting the sustainable use of the park's numerous resources to actualize the potential value non-consumptively through ecotourism is hereby recommended.
\end{abstract}

Keywords: attraction, conservation ecotourism, national park, rainforest

\section{Introduction}

Tourism is both leisure and a self-indulgent pursuit. It is a global phenomenon that has experienced rapid growth in the developing countries of the world. Smith (2004) 
noted that tourism and travel have been part of the human experience for millennia, describing it as a form of movement that characterizes Homo sapiens. Ecotourism is a type of specialty travel incorporating a diverse array of activities and tourism type, from bird watching, game viewing, scientific study, photography, diving, trekking, to regeneration of damaged ecosystems. The International Ecotourism Society defined ecotourism as "responsible travel to natural areas that conserves the environment, sustains the well-being of the local people, and involves interpretation and education" (TIES 2015). It has been able to capitalize on the increased motivation to experience and preserve natural environments (Diamantis 2004). Tourism industry has wholly embraced ecotourism, and its global importance has been recognized by the UN through the declaration of the year 2002 as the "International Year of Ecotourism"

The concern of tourists for environmental issues has increased, and ecotourism has become a known sector of tourism that should be developed (Holden 2003), because of its strategy of understanding nature and imparting conservation measure on biodiversity. Biodiversity is the backbone of the ecotourism industry. The role of biodiversity in tourism ranges from biodiversity as an attraction (i.e., many wildlife are focal species), resources for consumptive goods (i.e., culinary), natural components to support ecological services (i.e., pollination), to aesthetics (i.e., ornamental plants) (Higginbottom 2004, Newsome et al. 2012). Biodiversity, however, is not a tourist attraction unless its tourism potential value is converted and actualized as objects which are able to attract tourists (Drumm and Moore 2002). The success of tourism attraction, therefore, depends on the ability of tourism planners and managers to actualize the potential value of biodiversity as a tourist attraction (Luchman 2017). Ecotourism is seen as an interfacing of conservation concerns and tourism interest. Essentially, ecotourism derives its attraction from a combination of groups to be made from marketing a product that exists in its natural state in a specific geographic location and the potential to make such ecologically, economically, and socially sustainable (Ijeoma 2007).

The National Park divisions at Oban and Okwango in Cross River State, Nigeria is a wildlife park suitable for ecotourism. The Cross River National Park (CRNP) is an important ecological gene pool containing one of the oldest rainforests in Africa. Because of its critical conservation status, it has been designated as one of the $25 \mathrm{UN}$ biodiversity hot spots in the world (CRNP 2010). The forest resources represent a tourism haven capable of instilling conservation consciousness in the mind of the tourists that patronize the park. Therefore, adequate knowledge of the occurrence of the tourism products in an ecosystem is vital to enhance its effective marketing, increased patronage and sustainable use through ecotourism. Despite the enviable potential Nigeria is endowed with, the country has remained an unpopular tourist destination. Therefore, this study was conducted to identify the park's core tourist attractions, to determine the potential recreation activities of ecotourism, to assess the fauna and flora resources of CRNP forest as a draw for ecotourism and to promote argument for its successful conservation and sustainable use. This will enhance 
effective decision-making in the competing economic, social and environmental demands of sustainable development in Nigeria.

\section{Material and Methods}

\subsection{Study area}

Cross River National Park (CRNP) is located in Cross River State between longitudes $5^{\circ} .05^{\prime}-6^{\circ} .29^{\prime} \mathrm{N}$ and latitudes $8^{\circ} .15^{\prime}-90.30^{\prime} \mathrm{E}$ in Nigeria (Figure 1). It covers an area of approximately $4000 \mathrm{~km}^{2}$, divided into two non-contiguous divisions - the Oban hills in the Southern part covering $3000 \mathrm{~km}^{2}$, and the Okwangwo division in the Northern part covering $1000 \mathrm{~km}^{2}$ of primary moist rainforest ecosystem in the Northern and Central parts, and montane mosaic on the Obudu plateau (CRNP 2010).

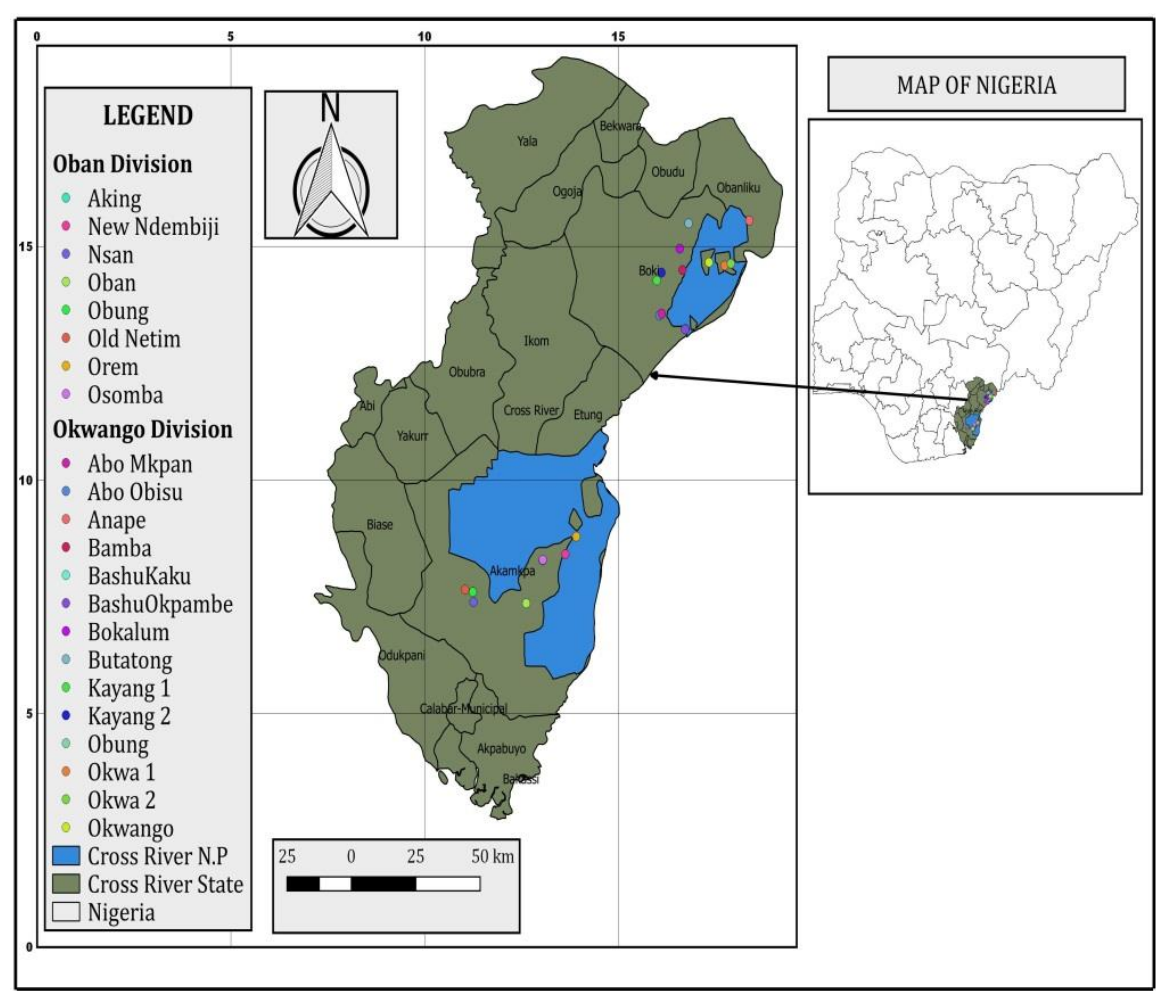

Fig. 1: Oban and Okwangwo divisions of Cross River National Park with Support Zone Communities 
The terrain is rough, and elevation rises from the river valleys to over $1000 \mathrm{~m}$ in mountainous areas. The Park has a tropical climate characterized by a rainy season between April and November. The annual rainfall ranges between $2000 \mathrm{~mm}$ to 3000 $\mathrm{mm}$; relative humidity in and around the park range from 80-90\%. The temperature rarely falls below $19^{\circ} \mathrm{C}$ with an annual mean of $27^{\circ} \mathrm{C}$ (Udoidung et al. 2007). Many ecosystems including the tropical rainforest and mangrove forest occur in Cross River State. The geographical location of the state favours ecotourism as the state is accessible by air through the Calabar Airport, by sea through the Calabar Sea Port and by road through the trunk roads from Akwa Ibom, Ebonyi and Abia States (Udoidung et al. 2007). The ecology of the state has made it a renowned wildlife sanctuary and the government has made substantial investment to develop its tourism potentials across the state in order to realize its aspiration of making the state the flagship of tourism in the country. The rich cultural heritage and strategic coastal location are great impetus for the rapidly developing culture and business tourism in the state.

\subsection{Data collection and analysis}

Ecotourism resources available in the park were carefully observed and assessed. The Global Positioning System (GPS) surveying method was used in the mapping of ecotourism resources in the study area (Shrestha 2006). Relevant pictures that depict specific and exciting characteristic features of interest were taken using digital Camera-Samsung zoom lens 5x (14.2 Megapixels) and GPS (GPS- 550 Magellan) for the geographic coordinates reading. Fauna resources were identified along the existing $5 \mathrm{Km}$ transects, trails and Jeep tracks within the park. Observations were recorded between 6.30 am to $11.00 \mathrm{am}$ in the morning and 4.00 to $6.30 \mathrm{pm}$ in the evening three days a week to identify the fauna species. Twenty-four days of observation were carried out in February (Dry season) and August (Rainy season), 2012 , making up 12 days in each season. Spoors such as faecal droppings, footprints spines, calls, and trails were recorded and used to determine the presence of the animals in the park in addition to the direct observations. There are no recent studies so these data will provide some baseline information.

Quadrat method was used for the vegetation assessment of Oban and Okwango divisions of the park. The method adopted by FAO (2009) was modified and a total of 15 plots with $50 \mathrm{~m} \times 20 \mathrm{~m}$ quadrant size each were established on three lines transversed evenly along the existing Jeep track within the study area. Five plots were marked on each line at $10 \mathrm{~km}$ interval. Identification of tree species within each plot was carried out. All identified trees were allocated to families and the number of species was obtained for the diversity classification of tree species in each family using Keay (1989). The total density was calculated as well as the diversity and species evenness. Species relative density (RD) and relative frequency (RF) of each site were computed using equations 1 and 2 . Species diversity index was calculated 
using Shannon-Wiener diversity index (eq. 3), while Shannon's equitability index $\left(E_{H}\right)$ (eq. 4) was adopted for estimating species evenness.

\section{Species Relative Density (RD):}

This refers to the number of individuals of a given species divided by the total number of individuals of all species.

$$
\mathrm{RD}=\left[\frac{\mathrm{ni}}{\mathrm{N}}\right] \mathrm{X} 100
$$

where, $\mathrm{RD}=$ relative density, $\mathrm{n}_{\mathrm{i}}=$ number of individual species $\mathrm{I}$, and $\mathrm{N}=$ total number of individual in the entire population.

\section{Relative Frequency (RF):}

RF was obtained using the formula given by Oduwaiye et al. (2002).

$$
\mathrm{RF}=\frac{\sum \mathrm{FiX} 100}{\mathrm{Fn}}
$$

where, $\mathrm{RF}=$ relative frequency, $\mathrm{Fi}=$ number of plot where species was found, and $\mathrm{Fn}$ $=$ total frequency of all species.

\section{Shannon-Wiener Diversity Index $\left(\mathbf{H}^{\mathrm{I}}\right)$ :}

The equation given by Price (1997) was adopted.

$$
\mathrm{H}^{1}=\sum_{\mathrm{i}=1}^{\mathrm{s}} \mathrm{Pi} \ln \mathrm{Pi}
$$

where, $\mathrm{S}=$ total number of species in the community, $\mathrm{Pi}=$ proportion of a species to the total number of plant in the community, $l n=$ natural logarithm.

\section{Species Evenness Index $\left(\mathbf{E}_{\mathbf{H}}\right)$ :}

In each forest community, species evenness was determined using Shannon's Equitability $\left(\mathrm{E}_{\mathrm{H}}\right)$.

$$
\mathrm{E}_{\mathrm{H}}=\frac{\mathrm{H}}{\mathrm{Hmax}}=\frac{\sum_{\mathrm{i}=1}^{\mathrm{S}} \mathrm{piln}(\mathrm{pi})}{\ln (\mathrm{S})}
$$




\section{Results}

\subsection{Ecotourism resources in Cross River National Park}

Prominent recreational activities that can be conducted in the Cross River National Park (CRNP) are hiking in the rainforest vegetation, mountaineering, water recreation in the natural water body (e.g. Kwa river), game viewing, bird watching, botanical garden tour and visit the natural history museum as presented in Table 1 . The topography presents very interesting sceneries for ecotourism activities and harbors rare species of animals such as the critically endangered Cross River Gorilla (Gorilla gorilla diehli), and the endangered Chimpanzee (Pan troglodytes). Village tours can provide visitors the opportunity to explore the way of life of the local communities.

Table 1: Ecotourism attractions in Cross River National Park, Nigeria.

\begin{tabular}{ll}
\hline Attractions & Location \\
\hline Wilderness hiking & The entire park \\
$\begin{array}{l}\text { Game viewing/ bird watching } \\
\text { Mountain viewing/ climbing }\end{array}$ & $\begin{array}{l}\text { The entire park } \\
\text { Park rugged terrain rising from } 100 \mathrm{~m} \text { in the river valleys to } \\
\text { over 1000m in the mountains Mbe Mountain }\end{array}$ \\
Forest camping & The entire park \\
Rock formation & Bat caves in Oban division \\
Village tours & 105 Support Zone communities of CRNP \\
Tour of botanical garden & Okwangwo Division \\
Water recreation & Natural water pool - kwa- river, Bemi river, Oyi river and \\
Natural History Museum & Okon river \\
\hline
\end{tabular}

CRNP is endowed with biodiversity resources, which serve as a major source to attract ecotourists to this area. Fifteen wild animal species belonging to 9 families were observed (Table 2) and a total of 81 tree species belonging to 26 families were identified in Oban and Okwango divisions of CRNP (Tables 3 and 4). The diversity index $\left(\mathrm{H}^{\prime}\right)$ and equitability (evenness) $\left(\mathrm{E}_{\mathrm{H}}\right)$ for Oban Division is 3.88 and 0.62 and for Okwango division $H$ is 3.84 and $\left(\mathrm{E}_{\mathrm{H}}\right) 0.53$, respectively (Table 5).

According to the Redlist Conservation Status in 2015, the animals encountered included endangered Drill monkey (Mandrillus leucophaecus) vulnerable Elephant (Loxodonta africana cyclotis) near threatened Bat (Eidolon helvum), and others were at the least concerned status. The endemic Bare-headed rock fowl Picarthates oreas 
and the Cameroonian vine, Ancistrocladus korupensis are "ecostars" and "a must see" that warrant a visit to the park.

Table 2: Fauna species encountered in Cross River National Park, Nigeria.

\begin{tabular}{|c|c|c|c|c|c|c|}
\hline Family & $\begin{array}{l}\text { Common } \\
\text { Name }\end{array}$ & Scientific Name & Evidence & Remark & $\begin{array}{l}\text { IUCN } \\
\text { Status * }\end{array}$ & $\begin{array}{l}\text { Population } \\
\text { Trend }\end{array}$ \\
\hline Bovidae & $\begin{array}{l}\text { Red flanked } \\
\text { duiker }\end{array}$ & $\begin{array}{l}\text { Cephalophus } \\
\text { rufilatus }\end{array}$ & $\begin{array}{l}\text { Faecal } \\
\text { dropping }\end{array}$ & Fresh & $\mathrm{LC}$ & Decreasing \\
\hline Bovidae & Blue duiker & $\begin{array}{c}\text { Cephalophus } \\
\text { monticola }\end{array}$ & Footprint/ trail & Fresh & LC & Stable \\
\hline Bovidae & Bush buck & Tragelaphus scriptus & Footprint & Fresh & $\mathrm{LC}$ & Stable \\
\hline Hystricidae & Porcupine & Altherurus africanus & Footprint/ trail & Fresh & $\mathrm{LC}$ & Unknown \\
\hline Suidae & $\begin{array}{l}\text { Red River } \\
\text { hog }\end{array}$ & $\begin{array}{l}\text { Potamochoerus } \\
\text { porcus }\end{array}$ & Footprint & Fresh & $\mathrm{LC}$ & Decreasing \\
\hline Sciuridae & $\begin{array}{l}\text { Giant tree } \\
\text { squirrel }\end{array}$ & Protoxerus stangeri & Sighting & On a tree & $\mathrm{LC}$ & Unknown \\
\hline Sciuridae & $\begin{array}{l}\text { Ground } \\
\text { squirrel }\end{array}$ & Xerus erythropus & Sighting & $\begin{array}{l}\text { Crossing } \\
\text { transect line }\end{array}$ & $\mathrm{LC}$ & Stable \\
\hline Elephantidae & $\begin{array}{l}\text { Forest } \\
\text { elephant }\end{array}$ & $\begin{array}{l}\text { Loxodonta africana } \\
\text { cyclotis }\end{array}$ & $\begin{array}{l}\text { Footprint/ } \\
\text { feeding trail/ } \\
\text { dung }\end{array}$ & $\begin{array}{l}\text { Dung about } \\
2 \text { weeks old }\end{array}$ & V & Increasing \\
\hline Viverridae & Palm Civet & Viverra civetta & Sighting & $\begin{array}{l}\text { individual on } \\
\text { tree }\end{array}$ & $\mathrm{LC}$ & Decreasing \\
\hline Viverridae & Mongoose & Ichneumia albicauda & Footprint & $\begin{array}{l}\text { More than } \\
10 \text { sighting }\end{array}$ & $\mathrm{LC}$ & Stable \\
\hline Cercopithecidae & Drill Monkey & $\begin{array}{l}\text { Mandrillus } \\
\quad \text { leucophaeus }\end{array}$ & $\begin{array}{l}\text { Sighting/ } \\
\text { Vocalisation }\end{array}$ & $\begin{array}{l}\text { About } 15 \\
\text { individuals } \\
\text { on a tree } \\
\text { feeding on } \\
\text { fruit }\end{array}$ & EN & Unknown \\
\hline Cercopithecidae & $\begin{array}{l}\text { Mona } \\
\text { monkey }\end{array}$ & Cercopithecus mona & $\begin{array}{l}\text { Sighting/ } \\
\text { Vocalisation }\end{array}$ & 5 individuals & $\mathrm{LC}$ & Unknown \\
\hline Cercopithecidae & $\begin{array}{c}\text { Tantalus } \\
\text { monkey }\end{array}$ & $\begin{array}{l}\text { Cercopithecus } \\
\text { aethiops }\end{array}$ & Sighting & 1 individual & $\mathrm{LC}$ & Stable \\
\hline Capitonidae & $\begin{array}{l}\text { Yellow- } \\
\text { spotted } \\
\text { barbet }\end{array}$ & $\begin{array}{l}\text { Buccanodon } \\
\text { duchaillui }\end{array}$ & Sighting & 1 individual & $\mathrm{LC}$ & Stable \\
\hline Pteropodidae & Fruit Bats & Eidolon helvum & Sighting & $\begin{array}{l}\text { More than } \\
20 \text { sighted } \\
\text { at Erokut } \\
\text { rock }\end{array}$ & NT & Decreasing \\
\hline
\end{tabular}

*LC-Least Concern, V-Vulnerable, NT- Near Threatened, EN- Endangered 
Table 3: Tree species observed in Oban Division of Cross River National Park.

\begin{tabular}{|c|c|c|c|c|c|}
\hline Species & Family & $\begin{array}{c}\text { IUCN } \\
\text { Status \# }\end{array}$ & $\begin{array}{l}\text { Density } \\
(\mathrm{Ha}-1)\end{array}$ & $\begin{array}{l}\text { Relative } \\
\text { Density }\end{array}$ & $\mathrm{pi}^{*} \ln (\mathrm{pi})$ \\
\hline Allanblackia floribunda & Guttiferae & VU & 5 & 0.581395 & -0.02993 \\
\hline Alstonia congensis & Apocynaceae & $\mathrm{LC}$ & 44 & 1.98915 & -0.08204 \\
\hline Antrocaryon micraster & Anacardiaceae & VU & 8 & 0.180832 & -0.0121 \\
\hline Anonidium mannii & Annonaceae & $\mathrm{LC}$ & 12 & 0.542495 & -0.02993 \\
\hline Antiaris toxicaria & Moraceae & $\mathrm{LC}$ & 5 & 0.361664 & -0.02152 \\
\hline Anthocleista vogelii & Apocynaceae & $\mathrm{LC}$ & 5 & 0.180832 & -0.0121 \\
\hline Baphia nitida & Fabaceae & $\mathrm{LC}$ & 24 & 0.542495 & -0.02993 \\
\hline Bridelia micrantha & Euphorbiaceae & $\mathrm{LC}$ & 40 & 2.712477 & -0.10285 \\
\hline Brenania brelie & Leguminosae & $\mathrm{LC}$ & 2 & 0.180832 & -0.0121 \\
\hline Carapa procera & Meliaceae & $\mathrm{LC}$ & 22 & 2.531646 & -0.09787 \\
\hline Chrysophyllum albidum & Sapotaceae & NT & 5 & 0.361664 & -0.02152 \\
\hline Christiana africana & Tiliaceae & $\mathrm{LC}$ & 16 & 0.180832 & -0.0121 \\
\hline Cleistopholis patens & Annonaceae & $\mathrm{LC}$ & 8 & 0.361664 & -0.02152 \\
\hline Cola acuminate & Sterculiaceae & $\mathrm{LC}$ & 64 & 0.723327 & -0.03767 \\
\hline Corynanthe pachyceras & Rubiaceae & $\mathrm{LC}$ & 64 & 0.723327 & -0.03767 \\
\hline Coula edulis & Olacaceae & $\mathrm{LC}$ & 154 & 5.244123 & -0.16179 \\
\hline Cylicodiscus gabunensis & Leguminosae & $\mathrm{LC}$ & 32 & 0.361664 & -0.02152 \\
\hline Distemonanthus benthamianus & Olacaceae & $\mathrm{LC}$ & 34 & 3.435805 & -0.12157 \\
\hline Dialium guineens & Leguminosae & $\mathrm{LC}$ & 192 & 2.169982 & -0.08747 \\
\hline Desplatsia dewevrei & Tiliaceae & $\mathrm{LC}$ & 96 & 2.169982 & -0.08747 \\
\hline Pycnanthus angolensis & Myristicaceae & $\mathrm{LC}$ & 16 & 0.180832 & -0.0121 \\
\hline Dacryodes edulis & Burseraceae & NL & 32 & 0.361664 & -0.02152 \\
\hline Diospyros mespiliformis & Ebenaceae & $\mathrm{LC}$ & 13 & 0.723327 & -0.03767 \\
\hline Desplatsia dewevrei & Tiliaceae & $\mathrm{LC}$ & 6 & 0.361664 & -0.02152 \\
\hline Diospyros suaveolens & Ebenaceae & NL & 5 & 0.361664 & -0.02152 \\
\hline Diospyros zenkeri & Ebenaceae & $\mathrm{LC}$ & 84 & 3.797468 & -0.1303 \\
\hline Enantia chlorantha & Annonaceae & $\mathrm{LC}$ & 21 & 0.723327 & -0.03767 \\
\hline Entandrophragma cylindricum & Meliaceae & VU & 11 & 0.361664 & -0.02152 \\
\hline Ficus capensis & Moraceae & NL & 32 & 0.723327 & -0.03767 \\
\hline Funtumia elastica & Apocynaceae & $\mathrm{LC}$ & 48 & 0.542495 & -0.02993 \\
\hline Garcinia mannii & Guttiferae & VU & 88 & 1.98915 & -0.08204 \\
\hline Garcinia staudtii & Guttiferae & VU & 32 & 0.361664 & -0.02152 \\
\hline Guibourtia ehie & Leguminosae & $\mathrm{LC}$ & 16 & 0.180832 & -0.0121 \\
\hline Guarea glomerulata & Meliaceae & NL & 21 & 0.723327 & -0.03767 \\
\hline Guarea thompsonii & Meliaceae & VU & 16 & 0.361664 & -0.02152 \\
\hline Hannoa klaineana & Simaroubaceae & NL & 112 & 1.265823 & -0.05834 \\
\hline Hunteria umbellata & Apocynaceae & $\mathrm{LC}$ & 112 & 1.265823 & -0.05834 \\
\hline Distemonanthus benthamianus & Leguminosae & $\mathrm{LC}$ & 48 & 0.542495 & -0.02993 \\
\hline Irvingia gabonensis & Irvingaceae & NT & 32 & 0.361664 & -0.02152 \\
\hline Khaya ivorensis & Meliaceae & VU & 2 & 0.180832 & -0.0121 \\
\hline Lophira alata & Ochnaceae & VU & 8 & 0.361664 & -0.02152 \\
\hline Maesobotrya barteri & Moraceae & $\mathrm{LC}$ & 88 & 1.98915 & -0.08204 \\
\hline Microdesmis puberula & Euphorbiaceae & NL & 12 & 0.542495 & -0.02993 \\
\hline Milicia excelsa & Moraceae & NT & 2 & 0.180832 & -0.0121 \\
\hline Millettia griffoniana & Leguminosae & $\mathrm{LC}$ & 64 & 2.169982 & -0.08747 \\
\hline Musanga cecropioides & Moraceae & $\mathrm{LC}$ & 52 & 2.350814 & -0.09274 \\
\hline Neoboutonia glabrescens & Euphorbiaceae & NT & 5 & 0.361664 & -0.02152 \\
\hline Nesogordonia papaverifera & Sterculiaceae & VU & 8 & 0.180832 & -0.0121 \\
\hline Newbouldia laevis & Bignoniaceae & NL & 8 & 0.180832 & -0.0121 \\
\hline
\end{tabular}


Table 3. Continued

\begin{tabular}{|c|c|c|c|c|c|}
\hline Species & Family & $\begin{array}{c}\text { IUCN } \\
\text { Status \# }\end{array}$ & $\begin{array}{r}\text { Density } \\
(\mathrm{Ha}-1)\end{array}$ & $\begin{array}{l}\text { Relative } \\
\text { Density }\end{array}$ & $\mathrm{pi} * \ln (\mathrm{pi})$ \\
\hline Octoknema affinis & Octoknemaceae & NL & 11 & 0.723327 & -0.03767 \\
\hline Omphalocarpum procerum & Sapotaceae & $\mathrm{LC}$ & 2 & 0.180832 & -0.0121 \\
\hline Ongokea gore & Olacaceae & $\mathrm{LC}$ & 3.2 & 0.361664 & -0.02152 \\
\hline Ouratea calophylla & Ochnaceae & NL & 5 & 0.361664 & -0.02152 \\
\hline Acacia albida & Leguminosae & $\mathrm{LC}$ & 60 & 0.180832 & -0.10285 \\
\hline Parkia bicolor & Leguminosae & $\mathrm{LC}$ & 112 & 2.712477 & -0.05834 \\
\hline Pentaclethra macrophylla & Leguminosae & $\mathrm{LC}$ & 48 & 1.265823 & -0.02993 \\
\hline Piptadeniastrum africanum & Leguminosae & $\mathrm{LC}$ & 5 & 0.542495 & -0.0121 \\
\hline Poga oleosa & Anisophylleaceae & $\mathrm{LC}$ & 16 & 0.180832 & -0.0121 \\
\hline Prosopis africana & Leguminosae & $\mathrm{LC}$ & 18 & 0.180832 & -0.07642 \\
\hline Pycnanthus angolensis & Myristicaceae & NL & 32 & 1.808318 & -0.02152 \\
\hline Rauvolfia mannii & Apocynaceae & $\mathrm{LC}$ & 32 & 0.723327 & -0.03767 \\
\hline Rauvolfia vomitoria & Apocynaceae & $\mathrm{LC}$ & 128 & 1.446655 & -0.0646 \\
\hline Rothmannia hispida & Rubiaceae & $\mathrm{LC}$ & 96 & 1.084991 & -0.05179 \\
\hline Amphimas pterocarpoids & Leguminosae & $\mathrm{LC}$ & 3 & 0.180832 & -0.0121 \\
\hline Spathodea campanulate & Bignoniaceae & $\mathrm{LC}$ & 3 & 0.180832 & -0.0121 \\
\hline Staudtia stipitate & Myristicaceae & $\mathrm{LC}$ & 24 & 1.627486 & -0.07062 \\
\hline Dacryodes edulis & Burseraceae & NL & 4 & 0.180832 & -0.0121 \\
\hline Sterculia oblonga & Sterculiaceae & $\mathrm{LC}$ & 16 & 0.542495 & -0.02993 \\
\hline Sterculia rhinopetala & Sterculiaceae & $\mathrm{LC}$ & 11 & 0.361664 & -0.02152 \\
\hline Sterculia tragacantha & Sterculiaceae & $\mathrm{LC}$ & 32 & 0.723327 & -0.03767 \\
\hline Strombosia grandifolia & Olacaceae & NL & 448 & 5.063291 & -0.15812 \\
\hline Strombosia pustulata & Olacaceae & $\mathrm{LC}$ & 368 & 8.318264 & -0.21551 \\
\hline Tabernaemontana pachysiphon & Apocynaceae & $\mathrm{LC}$ & 80 & 0.904159 & -0.04493 \\
\hline Trichilia lanata & Meliaceae & $\mathrm{LC}$ & 112 & 1.265823 & -0.05834 \\
\hline Trilepisium madagascariense & Moraceae & CR & 48 & 1.627486 & -0.07062 \\
\hline Uapaca staudtii & Euphorbiaceae & $\mathrm{LC}$ & 48 & 1.084991 & -0.05179 \\
\hline Uapaca togoensis & Euphorbiaceae & $\mathrm{LC}$ & 64 & 0.723327 & -0.03767 \\
\hline Vitex doniana & Verbenaceae & $\mathrm{LC}$ & 32 & 0.361664 & -0.02152 \\
\hline Xylopia aethiopica & Annonaceae & $\mathrm{LC}$ & 144 & 1.627486 & -0.07062 \\
\hline Zanthoxylum zanthoxyloides & Rutaceae & $\mathrm{EN}$ & 64 & 0.723327 & $\begin{array}{l}-0.03767 \\
3.88336\end{array}$ \\
\hline
\end{tabular}

*LS (Least Concerned), V (Vulnerable), NT (Near Threatened), NL (Not Listed), E (endangered), CR (Critically Endangered)

A total of 80 tree species belonging to 25 families was identified in Oban division while 78 species belonging to 22 families were recorded in Okwango division. Leguminosae (12) and Apocynaceae (10) were the most dominant families in Oban and Okwango divisions respectively. Other dominant families in both divisions were Moraceae, Olacaceae, and Guttiferae. The slight variation in the diversity index signified that Oban division is more diverse in woody tree species than Okwango division. Notable amongst the flora species are the critically endangered (Trilepisium madagascariense), endangered (Zanthoxylum zanthoxyloides), Near threatened (Irvingia gabonensis, Neoboutonia glabrescens, Milicia excels, Chrysophyllum albidum) and Vulnerable (Nesogordonia papaverifera, Lophira alata, Khaya 
ivorensis, Guarea thompsonii, Garcinia mannii, Garcinia staudtii, Entandrophragma cylindricum, Antrocaryon micraster, Allanblackia floribunda).

Table 4: Tree species observed in Okwango Division of Cross River National Park.

\begin{tabular}{|c|c|c|c|c|c|}
\hline Species & Family & $\begin{array}{c}\text { IUCN } \\
\text { Status \# }\end{array}$ & $\begin{array}{r}\text { Density } \\
(\mathrm{Ha}-1)\end{array}$ & $\begin{array}{l}\text { Relative } \\
\text { Density }\end{array}$ & $\mathrm{pi}^{*} \ln (\mathrm{pi})$ \\
\hline Acacia albida & Apocynaceae & $\mathrm{LC}$ & 2 & 0.246914 & -0.01218 \\
\hline Allanblackia floribunda & Guttiferae & VU & 7 & 0.740741 & -0.03012 \\
\hline Alstonia congensis & Apocynaceae & $\mathrm{LC}$ & 59 & 2.716049 & -0.08251 \\
\hline Amphimas pterocarpoides & Apocynaceae & $\mathrm{LC}$ & 6 & 0.493827 & -0.02166 \\
\hline Anonidium mannii & Annonaceae & $\mathrm{LC}$ & 8 & 0.740741 & -0.03012 \\
\hline Anthocleista vogelii & Apocynaceae & $\mathrm{LC}$ & 5 & 0.246914 & -0.01218 \\
\hline Antiaris toxicaria & Moraceae & $\mathrm{LC}$ & 6 & 0.493827 & -0.02166 \\
\hline Antrocaryon micraster & Apocynaceae & VU & 176 & 8.148148 & -0.17672 \\
\hline Baphia nitida & Fabaceae & $\mathrm{LC}$ & 24 & 0.740741 & -0.03012 \\
\hline Brenania brelie & Fabaceae & NL & 16 & 0.246914 & -0.01218 \\
\hline Bridelia micrantha & Fabaceae & $\mathrm{LC}$ & 80 & 3.703704 & -0.10343 \\
\hline Carapa procera & Meliaceae & $\mathrm{LC}$ & 45 & 3.45679 & -0.09842 \\
\hline Christiana africana & Olacaceae & $\mathrm{LC}$ & 3 & 0.246914 & -0.01218 \\
\hline Chrysophyllum albidum & Sapotaceae & NT & 3 & 66.66667 & -0.02166 \\
\hline Cleistopholis patens & Annonaceae & $\mathrm{LC}$ & 11 & 0.493827 & -0.02166 \\
\hline Sterculia oblonga & Sterculiaceae & $\mathrm{LC}$ & 4 & 0.987654 & -0.03791 \\
\hline Staudtia stipitate & Olacaceae & $\mathrm{LC}$ & 4 & 0.987654 & -0.03791 \\
\hline Coula edulis & Olacaceae & $\mathrm{LC}$ & 240 & 7.407407 & -0.16624 \\
\hline Cylicodiscus gabunensis & Olacaceae & $\mathrm{LC}$ & 16 & 0.493827 & -0.02166 \\
\hline Dacryodes edulis & Burseraceae & NL & 16 & 0.493827 & -0.02166 \\
\hline Desplatsia dewevrei & Tiliaceae & $\mathrm{LC}$ & 5 & 0.493827 & -0.02166 \\
\hline Dialium guineense & Leguminosae & $\mathrm{LC}$ & 96 & 2.962963 & -0.08797 \\
\hline Diospyros mespiliformis & Ebenaceae & $\mathrm{LC}$ & 21 & 0.987654 & -0.03791 \\
\hline Diospyros suaveolens & Ebenaceae & NL & 16 & 0.493827 & -0.02166 \\
\hline Diospyros zenkeri & Ebenaceae & $\mathrm{LC}$ & 168 & 5.185185 & -0.131 \\
\hline Distemonanthus benthamianus & Leguminosae & $\mathrm{LC}$ & 304 & 4.691358 & -0.12223 \\
\hline Drypetes chevalieri & Euphorbiaceae & NL & 64 & 2.962963 & -0.08797 \\
\hline Enantia chlorantha & Annonaceae & $\mathrm{LC}$ & 32 & 0.987654 & -0.03791 \\
\hline Entandrophragma cylindricum & Meliaceae & VU & 32 & 0.493827 & -0.02166 \\
\hline Ficus capensis & Moraceae & NL & 64 & 0.987654 & -0.03791 \\
\hline Funtumia elastica & Apocynaceae & $\mathrm{LC}$ & 10 & 0.740741 & -0.03012 \\
\hline Garcinia staudtii & Guttiferae & VU & 16 & 0.493827 & -0.02166 \\
\hline Garcinia mannii & Guttiferae & VU & 88 & 2.716049 & -0.08251 \\
\hline Guarea glomerulata & Guttiferae & NL & 96 & 1.481481 & -0.05211 \\
\hline Guarea thompsonii & Meliaceae & VU & 32 & 0.493827 & -0.02166 \\
\hline Guibourtia ehie & Guttiferae & $\mathrm{LC}$ & 8 & 0.246914 & -0.01218 \\
\hline Hannoa klaineana & Simaroubaceae & NL & 112 & 1.728395 & -0.05869 \\
\hline Hunteria umbellate & Guttiferae & $\mathrm{LC}$ & 112 & 1.728395 & -0.05869 \\
\hline Irvingia gabonensis & Irvingiaceae & NT & 32 & 0.493827 & -0.02166 \\
\hline Khaya ivorensis & Meliaceae & VU & 16 & 0.246914 & -0.01218 \\
\hline Lophira alata & Ochnaceae & VU & 16 & 0.493827 & -0.02166 \\
\hline Maesobotrya barteri & Euphorbiaceae & $\mathrm{LC}$ & 176 & 2.716049 & -0.08251 \\
\hline Microdesmis puberula & Euphorbiaceae & NL & 16 & 0.740741 & -0.03012 \\
\hline Milicia excelsa & Moraceae & NT & 8 & 0.246914 & -0.01218 \\
\hline
\end{tabular}


Table 4. Continued

\begin{tabular}{|c|c|c|c|c|c|}
\hline Species & Family & $\begin{array}{l}\text { IUCN } \\
\text { Status \# }\end{array}$ & $\begin{array}{r}\text { Density } \\
(\mathrm{Ha}-1)\end{array}$ & $\begin{array}{l}\text { Relative } \\
\text { Density }\end{array}$ & $\mathrm{pi}^{*} \ln (\mathrm{pi})$ \\
\hline Millettia griffoniana & Leguminosae & LC & 96 & 2.962963 & -0.08797 \\
\hline Musanga cecropioides & Moraceae & $\mathrm{LC}$ & 208 & 3.209877 & -0.09327 \\
\hline Neoboutonia glabrescens & Moraceae & NT & 11 & 0.493827 & -0.02166 \\
\hline Nesogordonia papaverifera & Moraceae & VU & 8 & 0.246914 & -0.01218 \\
\hline Newbouldia laevis & Moraceae & NL & 16 & 0.246914 & -0.01218 \\
\hline Octoknema affinis & Olacaceae & NL & 64 & 0.987654 & -0.03791 \\
\hline Omphalocarpum procerum & Moraceae & $\mathrm{LC}$ & 3 & 0.246914 & -0.01218 \\
\hline Ongokea gore & Moraceae & $\mathrm{LC}$ & 16 & 0.493827 & -0.02166 \\
\hline Ouratea calophylla & Ochnaceae & NL & 16 & 0.493827 & -0.02166 \\
\hline Parinari macrophylla & Rosaceae & NL & 112 & 1.728395 & -0.05869 \\
\hline Parkia bicolor & Leguminosae & $\mathrm{LC}$ & 240 & 3.703704 & -0.10343 \\
\hline Pentaclethra macrophylla & Leguminosae & $\mathrm{LC}$ & 8 & 0.246914 & -0.01218 \\
\hline Piptadeniastrum africanum & Leguminosae & $\mathrm{LC}$ & 64 & 0.987654 & -0.03791 \\
\hline Amphima spterocarpoides & Apocynaceae & NL & 16 & 0.246914 & -0.01218 \\
\hline Prosopis africana & Leguminosae & $\mathrm{LC}$ & 16 & 0.246914 & -0.01218 \\
\hline Pycnanthus angolensis & Myristicaceae & NL & 176 & 2.716049 & -0.08251 \\
\hline Rauvolfia mannii & Apocynaceae & $\mathrm{LC}$ & 32 & 0.987654 & -0.03791 \\
\hline Rauvolfia vomitoria & Apocynaceae & $\mathrm{LC}$ & 128 & 1.975309 & -0.06498 \\
\hline Rothmannia hispida & Rubiaceae & $\mathrm{LC}$ & 32 & 1.481481 & -0.05211 \\
\hline Irvingia gabonensis & Irvingaceae & NT & 8 & 0.246914 & -0.01218 \\
\hline Staudtia stipitate & Myristicaceae & $\mathrm{LC}$ & 72 & 2.222222 & -0.07103 \\
\hline Sterculia oblonga & Sterculiaceae & $\mathrm{LC}$ & 48 & 0.740741 & -0.03012 \\
\hline Sterculia rhinopetala & Sterculiaceae & $\mathrm{LC}$ & 11 & 0.493827 & -0.02166 \\
\hline Sterculia tragacantha & Sterculiaceae & $\mathrm{LC}$ & 32 & 0.987654 & -0.03791 \\
\hline Strombosia grandifolia & Olacaceae & NL & 448 & 6.91358 & -0.15893 \\
\hline Strombosia pustulata & Olacaceae & $\mathrm{LC}$ & 736 & 11.35802 & -0.21649 \\
\hline Tabernaemontana pachysiphon & Apocynaceae & $\mathrm{LC}$ & 16 & 1.234568 & -0.0452 \\
\hline Trichilia lanata & Meliaceae & $\mathrm{LC}$ & 56 & 1.728395 & -0.05869 \\
\hline Trilepisium madagascariense & Moraceae & CR & 72 & 2.222222 & -0.07103 \\
\hline Uapaca staudtii & Euphorbiaceae & LC & 96 & 1.481481 & -0.05211 \\
\hline Uapaca togoensis & Euphorbiaceae & $\mathrm{LC}$ & 64 & 0.987654 & -0.03791 \\
\hline Vitex doniana & Verbenaceae & $\mathrm{LC}$ & 16 & 0.493827 & -0.02166 \\
\hline Xylopia aethiopica & Annonaceae & $\mathrm{LC}$ & 144 & 2.222222 & -0.07103 \\
\hline Zanthoxylum zanthoxyloides & Rutaceae & EN & 64 & 0.987654 & $\begin{array}{c}-0.03791 \\
3.83659\end{array}$ \\
\hline
\end{tabular}

Table 5: Summary of tree species diversity indices and distribution in Oban and Okwangwo Divisions of Cross River National Park (CRNP).

\begin{tabular}{lcccc}
\hline CRNP Division & $\begin{array}{l}\text { No. of tree } \\
\text { species }\end{array}$ & No. of families & $\begin{array}{c}\text { Shannon-Weiner diversity } \\
\text { index }\left(\mathrm{H}^{\prime}\right)\end{array}$ & $\begin{array}{c}\text { Species } \\
\text { evenness }\left(\mathrm{E}_{\mathrm{H}}\right)\end{array}$ \\
\hline Oban & 80 & 25 & 3.88 & 0.62 \\
Okwango & 78 & 22 & 3.84 & 0.53 \\
\hline
\end{tabular}




\section{Discussion}

Cross River National Park is a unique ecotourism site with its rich natural resources, geomorphological structure, rivers, climate and rich biological diversity. The core attractions of the park include wilderness hiking, game viewing, mountaineering, forest camping, rock formation, visits to the botanical garden, water recreation and village tours. Hiking in the rainforest vegetation was the prominent activity as the park provides wonderful wilderness experience with its dense canopy forests, consisting of tall trees with huge buttresses, the coolness of the environment, the freshness of the air and the sweet songs of the forest- dwelling birds. Its rich flora provides suitable habitats to many wild animals such as the Cross river gorilla, (Gorilla gorilla diehli) and the Chimpanzee (Pan troglodytes) and the bare-headed rock fowl (Picarthates oreas). Birds and monkey call audible in the forest would be of interest to ecotourists. This agrees with Drumm and More (2002) assertion about ecotourism attraction whether they are wildlife viewing possibilities or dramatic natural landscapes, tend to be found in protected natural areas. Moreover, Rajib and Jaba (2012) stated that natural environment was the major purpose of tourists' visit to Bangladesh.

The Erokut rock formation is a major attraction to tourist where bat watching is prominent. Bemi River (referred to as natural swimming pool) in Butatong, Okwango division and Kwa river (shallow water) in Erokut, Oban division are good ecotourism attractions where tourist can dive and swim. Plans are also on the ground by the park management to utilize the Kwa river for sport fishing and canoe riding for ecotourists. Other attractions include nature trail in Kayang at the foot of Mbe mountain, Natural History Museum in Oban division, Botanical Garden in Okwango division where Anceistrocladus kurupensis that was discovered to have high medicinal properties effective against HIV/AIDS can be found (Obot et al. 1996, CRNP 2008). Rock of Picathartes oreas (rock fowl) endemic bird species is found in Bashu (Okwango division) a distance of $2 \mathrm{~km}$ gentle slope from the community known as Bashu Bird Sanctuary. Traditional lifestyle and culture are important and attractive elements for ecotourists. Traditional architecture is an important element of the cultural landscape, with buildings of wood, bamboo and mud walls and grassthatched, high, pyramid-like roofs. The attribute of these resources in Cross River National Park makes the resources to be location specific for ecotourists and will have wide utility in the management of ecotourism in the park (Shrestha 2006, Srivastava and Anitha 2010).

Although fifteen wild animal species belonging to nine families were identified, the conservation status of the animals encountered show that six of the species are stable (Blue duiker Cephalophus rufilatus, Ground squirrel Xerus erythropus, Mongoose Ichneumia albicauda, Bush buck Tragelaphus scriptus, Tantalus monkey Cercopithecus aethiops and Yellow-spotted barbet Buccanodon duchaillui), four of the species' population is decreasing (Red flanked duiker Cephalophus rufilatus, Red 
river hog Potamochoerus porcus, Palm civet Viverra civetta, fruit bats Eidolon helvum) and the population change of four are unknown (Porcupine Altherurus africana, Giant tree squirrel Protoxerus stangeri, Drill monkey Mandrillus leucophaecus, and Mona monkey Cercopithecus mona). Elephant Loxodonta africana cyclotis is the only species whose population is increasing.

The family Leguminosae and Apocynacea were dominant in Oban and Okwangwo divisions respectively. Other dominant families in both divisions were Meliaceae, Euphorbiaceae, Moraceae, Olacaceae, Guttiferae and Sterculiaceae which are mostly found in Nigerian rainforests as reported by Adekunle and Olagoke (2008) and Onyekwelu et al. (2008). Moreover, the diversity index and equitability (evenness) for the two divisions slightly differ. This slight variation signified that Oban division is more diverse in woody tree species than Okwango division. These diversities were above 3.5 and fall within the general limit recommended by Kent and Coker (1992). The species diversities for the two divisions further elucidate Zakaria et al. (2016) assertion that tropical rainforests are the most diverse among the world ecosystem in vegetation structure and composition.

\section{Conclusions}

Cross River National Park with its natural resources of touristic significance is a powerful ecotourism destination. In spite of the tremendous resources available in CRNP, ecotourism development in the park is still at its infancy stage, its potentials to support activities like wilderness hiking, game viewing, bird watching, mountaineering, village tour, forest camping, water recreation where tourist can dive and swim, participate in sport fishing and canoe ride cannot be overemphasized. There was a presence of diverse biological resources that are endemic to this area including those declared threatened or endangered. The diversity assessment shows that the two divisions were able to conserve both fauna and flora diversity however Oban division was more diverse in woody tree species than Okwango division. Hence, the species richness of the park can be sustainably managed if the park is further prevented from anthropogenic effects occasioned by logging, hunting, and collection of forest products to improve the vegetation status and invariably enhance wildlife population in the park.

\section{Acknowledgements}

The Conservator General of Nigeria National Park Service is appreciated for granting approval to carry out this study in the park, Cross River National Park management is acknowledged for providing research unit staff during the field work and Dr. O.O. Sobola's contribution and insightful remarks is highly commended. Three anonymous reviewers are thanked for providing constructive feedback on the initial and revised versions. 


\section{References}

Adekunle VAJ, Olagoke AO. 2008. Diversity and biovolume of tree species in natural forest ecosystem in the Bitumen-producing areas of Ondo State, Nigeria. A baseline study. Biodiversity and Conservation 17: 2735-2755.

CRNP. 2008. Federal Ministry of Environment: National Park Service. Cross River National Park Annual Report, 1- 20pp.

CRNP. 2010. Federal Ministry of Environment: National Park Service. Cross River National Park Annual Report, 1- 39pp.

Diamantis D. 2004. Ecotourism Management: An Overview. In D. Diamantis (Ed.), Ecotourism-Management and Assessment. Thompson Learning, Belmont, CA. London. 1-26pp.

Drumm A, Moore A. 2002. Ecotourism Development: An Introduction to ecotourism Planning Volume1, The Nature Conservancy, USA. 85pp.

FAO. 2009. Food and Agriculture Organisation. National Forest Monitoring and Assessment. Manual for Local Level Assessment of Land Degradation, Sustainable Land Management and Livelihood, Land Degradation Assessment in Dry lands (LADA) Project, 39-66pp.

Higginbottom K. 2004. Wildlife tourism: impacts, management and planning. Wildlife tourism. Altona, Vic.: Common Ground Publishing for CRC for Sustainable Tourism. 277pp.

Holden A. 2003. Environment and Tourism, 1st Ed., Rutledge, England. 624pp.

Ijeomah HM. 2007. The impact of tourism on poverty levels of households in adjoining ecotourism destinations in plateau State. Ph.D Thesis. Department of Wildlife and Fisheries Management. University of Ibadan. Nigeria, 86-105pp.

Keay RWJ. 1989. Trees of Nigeria. A Revised Version of "Nigerian Trees" (Keay et al., 1964). Clarendon Press Oxford, 476pp.

Kent M, Coker P. 1992. Vegetation description and analysis. A Practical Approach, Belhaven Press, London, $363 p p$.

Luchman H. 2017. Managing biodiversity for a competitive ecotourism industry in tropical developing countries. New opportunities in biological fields AIP Conference Proceedings 1908, 030008; doi: $10.1063 / 1.5012708$.

Newsome D, Moore SA, Dowling R. 2012. Natural area tourism: Ecology, impacts, and management. Volume 58, Channel view publications.

Obot EA, Baker J, Ogar G. 1996. Biological Surveys in Cross River National Park as Contribution to Park Management. Cross River National Park (Okwango Division) Occasional Paper No. 2. Proceedings of Workshop on African Rainforest and the Conservation of Biodiveristy. Limbe Botanical Gardens, Cameroon, $17^{\text {th }}-24^{\text {th }}$ January, 1996, 3-4pp.

Oduwyaiye EA, Oyeleye B, Oguntala, AB. 2002. Species diversity and potentiality for forest regeneration in Okomu Sample plot in Abu JE, Oni, P. I. and Popoo;a. L. (eds) Forestry and Challnges of sustainable livelihood. In Proceeding of the $28^{\text {th }}$ Annual Conference of Forestry Assocciation of Nigeria, Akure.

Onyekwelu JC, Mosandl R, Stimm B. 2008. Tree species diversities and soil status of primary and degraded tropical rainforest ecosystems in south-western Nigeria. Journal of Tropical Forest Science 20: 193-204.

Price PW. 1997. Insect Ecology. John Wiley and Sons. ISBN: 978-0-471-16184-4 August 1997 888Pp.

Rajib KD, Jaba C. 2012. An evaluative study on tourism in Bangladesh. Research Journal of Finance and Accounting 2(1):17-28

Shrestha H. 2006. Globa Positioning Systems (GPS) and Geographical Information System (GIS) in Participatory Mapping of Community Forest in Nepal. EJISDC 25 (5):1-11.

Smith SLJ. 2004. The measurement of global tourism: Old debates, new consensus and continuing challenges. In: A. Lew, C.M. Hall and A. Williams (eds) A companion to Tourism. Oxford: Blackwell.

Srivastava V, Anitha D. 2010. Mapping of non-timber forest products using remote sensing and GIS. Tropical Ecology 51(1): 107-116

Udoidung NIG, Braide IE, Opara KN, Adie HA. 2007. Perstans filariasis in rural communities of lower Cross River Basin Nigeria. Parasitological Observation International Journal of Zoological Research 3:207-212.

Zakaria M, Rajpar MN, Ozdemir I, Rosli Z. 2016. Fauna Diversity in Tropical Rainforest: Threats from LandUse Change. Available from: https://www.intechopen.com/books. Retrieved on 22/01/2020. 